Horizons philosophiques

\title{
Les mutations du sacré et du symbolique dans les sociétés contemporaines : regard sur les festivités « rave »
}

\section{Pierre-Luc Lortie}

Volume 15, numéro 1, automne 2004

En quête du sujet

URI : https://id.erudit.org/iderudit/801278ar

DOI : https://doi.org/10.7202/801278ar

Aller au sommaire du numéro

Éditeur(s)

Collège Édouard-Montpetit

ISSN

1181-9227 (imprimé)

1920-2954 (numérique)

Découvrir la revue

Citer cet article

Lortie, P.-L. (2004). Les mutations du sacré et du symbolique dans les sociétés contemporaines : regard sur les festivités « rave ». Horizons philosophiques, 15(1), 79-90. https://doi.org/10.7202/801278ar d'utilisation que vous pouvez consulter en ligne. 


\section{Les mutations du sacré et du symbolique dans les sociétés contemporaines : regard sur les festivités «rave»}

Le siècle dernier fut marqué d'avancées scientifiques et technologiques prodigieuses dont les artisans eux-mêmes ont peine à mesurer la portée. Le développement croissant d'un tel secteur d'activités ne s'est toutefois pas réalisé de manière isolée; plusieurs autres sphères de la vie de l'homme moderne furent également bouleversées de manière fondamentale, à commencer par son rapport au sacré et au symbolique. Aujourd'hui, que l'on attribue le résultat de cette évolution à une continuation, rupture ou radicalisation des effets de la modernité, nos contemporains se voient néanmoins confrontés à un contexte de vie particulier où la construction de cohérence et de signification de l'être et de l'univers est dorénavant une entreprise individuelle.

Nouveau et inachevé, le phénomène du déplacement du sacré et du symbolique dans les sociétés contemporaines amène à modifier la manière d'appréhender les dimensions religieuses de l'homme. Depuis quelques années, plusieurs études, au Québec comme ailleurs, confirment en ce sens la présence et/ou l'émergence du religieux au sein de manifestations sociales jusque-là jugées profanes. Au sein de la culture actuelle et indistinctement d'une quelconque catégorisation du tissu social, le sacré tend effectivement à se déplacer, appelant à une interprétation plus large et plus signifiante de la part des sciences qui l'étudient. Le texte que le lecteur s'apprête à parcourir se donne donc comme objectif général de procéder à un survol des mutations du sacré et de la pensée symbolique dans le contexte particulier que connaissent les sociétés contemporaines. Pour ce faire, nous proposons ici une analyse des "communautés de sensation", plus communément nommées "raves". À la lumière de plusieurs caractéristiques inhérentes à ce phénomène, nous tenterons de dégager les éléments qui permettent de considérer ses festivités nocturnes comme étant des lieux où se manifeste une religiosité nouvelle.

Afin de se munir de bases conceptuelles pertinentes à l'étude de cette problématique spécifique, nous amorcerons l'analyse par l'ap- 
profondissement théorique du phénomène contemporain du déplacement du religieux de même que des principaux défis que cela soulève. Par la suite, en utilisant le concept de néo-tribalisme de Michel Maffesoli, en examinant l'analyse du rituel contemporain par Denis Jeffrey, l'anthropologie du corps et du risque proposé par David Le Breton, ainsi que l'ouvrage intitulé Technoritualité : Religiosité rave ${ }^{1}$ nous serons en mesure de dégager certains éléments qui feraient du phénomène rave une réponse à la quête de sens et de transcendance d'un certain nombre d'individus marqués par les présentes transformations sociales.

\section{Redéfinition de l'objet d'étude}

L'observation sociologique des phénomènes religieux contemporains pousse à admettre que si cette sphère d'activités a subi des transformations d'envergure au cours' de son passage dans la modernité, c'est le concept même du religieux qui doit alors être redéfini. En effet, peut-on encore parler avec autant d'assurance de la religion quand les productions religieuses de la modernité prennent des formes et des chemins qui divergent parfois nettement des grandes institutions traditionnelles? Peut-on même, à la lumière de nos connaissances, distinguer ce qui relève ou non du religieux? Enfin, peut-on seulement encore parler de religion en occident ou l'étude des croyances dans son ensemble ne permet-elle pas mieux de saisir l'actuel phénomène? Autant de questions qui relèvent, à mon sens, de l'angle par lequel le chercheur désire amorcer son analyse. Toutefois, il reste que lorsque l'on tente de recourir à une définition du religieux, celles proposées par les auteurs sont trop souvent restrictives au sens où elles ne possèdent pas la souplesse qu'exige l'étude de la "nouvelle demande de sens" propre à notre époque. Les transformations qui ont eu cours ces dernières années sur le plan des croyances et des productions religieuses prennent en effet des formes de plus en plus élargies. Autant par l'influence des représentations orientales que par celle de la science ou de la croissance personnelle et corporelle, les mouvements sont composés d'influences diverses. De plus, ajoutons à ce nouveau phénomène l'observation d'une succession d'actes ou de pratiques à la base de certains phénomènes et rassemblements qui prennent parfois des allures de grandes cérémonies et où la ferveur et les significations atteignent des niveaux à caractère incontestablement religieux. 
Face à ce constat, il faut donc se doter de définitions qui permettent - quitte à les ajuster ensuite suivant les nouveaux développements - de départager, parmi toutes les manifestations du social, celles qui relèvent d'une nouvelle production du religieux de celles qui n'en relèvent pas. Campiche et Bovay, dans l'ouvrage de Danièle Hervieu-Léger s'intitulant La religion pour mémoire, nous présentent une définition à la fois large et pertinente. On y définit la religion comme étant «tout ensemble de croyances et de pratiques, plus ou moins organisées, relatives à une réalité supraempirique transcendante» et associé à une série de fonctions : «intégration, identification, explication de l'expérience collective, réponse au caractère structurellement incertain de la vie individuelle et sociale ${ }^{2}$ ». Selon cette définition, le caractère religieux des croyances et pratiques pourra être considéré selon deux axes, soit par sa réalité transcendante et par sa valeur en tant que système d'explication et d'appartenance.

Bien que la définition du religieux proposée par Campiche et Bovay possède la souplesse nécessaire à l'étude des nouvelles formes de religiosité, une certaine rigueur méthodologique nous pousse à définir le concept de croyance. Ainsi, présentons la croyance comme l'ensemble des convictions d'une personne ou d'un groupe. Ce sont elles qui sous-tendent le sens et la cohérence de l'expérience vécue, ressentie par les hommes. En ce sens, les croyances ne sont pas directement mesurables et leur étude ne peut se faire qu'à partir de son objectivation, c'est-à-dire à partir de ses manifestations tangibles ${ }^{3}$. Le rôle et l'étude des croyances acquièrent donc leur signification lorsque nous les considérons comme moteur - de l'action humaine. Les choix d'un acteur ou d'une collectivité sont donc étroitement liés à ce concept. Invisibles, il reste néanmoins que les croyances seront davantage vécues que comprises par ceux qui les ont intégrées.

\section{Déplacement du religieux dans les sociétés contemporaines}

L'anthropologue Roger Bastide, dans un article de l'Encyclopédie Universalis, rendait compte en 1978 d'un phénomène qui, jusqu'à aujourd'hui, n'a cessé d'acquérir une grande pertinence. À cet égard il souligne :

Le religieux se déplace, plus qu'il ne disparaît, avec les cultes nouveaux nés de la sécularisation (...), avec leurs rites exta- 
tiques ou leurs pratiques cérémonielles (...). Le religieux n'est pas toujours dans ce que l'on appelle les religions; et, réciproquement, les religions sont souvent des rétrécissements, des institutions de défense contre le religieux, voire de simples annexes sentimentales d'un pur moralisme (... $)^{4}$.

Bastide, à l'instar de nombreux penseurs, soulignait un phénomène en émergence depuis la modernité, celui du déplacement du religieux dans des sphères d'activités sociales, phénomène qui, auparavant, n'était pas l'objet d'investigations de la part des sciences des religions. La prise de conscience d'une effervescence religieuse au sein de la modernité, contredisant du même coup les théories de la sécularisation largement répandues à cette époque, favorisa l'émergence et le développement, chez Luckmann comme chez d'autres, d'une théorie de la «religion invisible». Parmi les principaux champs et manifestations du sacré investigués aujourd'hui, on dénombre la question de l'identité nationale, de la sexualité, de la culture rock, des marques corporelles, des jeux de hasard et des groupes d'entraide pour alcooliques, comme autant de lieux où émerge l'exercice contemporain du religieux 5 . Manifestement, pour beaucoup, l'exercice du sacré et des croyances se voit dorénavant dispersé à travers l'ensemble du tissu social et mis en scène par des événements d'apparence souvent anodins. II n'empêche pas moins que l'étude d'un tel phénomène reste encore à ses débuts, tentant jour après jour de forger sa place et sa légitimité.

\section{Les communautés de sensation}

En amorçant l'étude du phénomène rave en tant que manifestation contemporaine du sacré, il est inévitable de trouver sur son parcours le discours d'une foule de pratiquants s'empressant de souligner la puissance de l'expérience rave, ainsi que du sentiment de transcendance qui s'en dégage. On peut aussi facilement dégager certaines analogies entre rave et religion lorsque, par exemple, l'expérience de ces manifestations est comparée à une messe, à une communion ou à une transe à caractère mystique. Le discjockey, quant à lui, peut être comparé à un chaman ou à un prêtre, prêchant la danse et l'effervescence au rythme de la musique ${ }^{6}$. Toutefois, bien que ces sentiments, partagés par beaucoup de raveurs, soulignent l'originalité et l'intensité de ces événements, ils ne suffisent pas pour justifier un déplacement réel de l'exercice du religieux en son sein. Néanmoins, avant d'approfondir certaines thèses par lesquelles il sera 
possible d'atteindre notre objectif, voyons quelles sont les caractéristiques particulières des rassemblements raves.

\section{Caractéristiques du mouvement rave}

Bien que difficilement quantifiable, le mouvement rave a ceci de particulier qu'il porte en lui un certain nombre de caractéristiques témoignant de la transformation des sociétés contemporaines, et ce, autant sur le plan politique, technologique, qu'esthétique. D'abord, contrairement à la contre-culture, à la mode punk ou rock, le mouvement rave n'est porteur d'aucun message politique ou idéologique et ne prétend à aucune réforme ou changement de l'ordre social. Néanmoins, il confirme, par sa simple existence, le besoin ressenti par les nouvelles générations de former, en marge de la société, des zones de "libre échange" de passions et de sensations avec, comme unique prétention, la création d'une expérience commune ${ }^{7}$. Serait-ce en réponse à un sentiment d'impuissance face à la chose politique ou à l'impression répandue chez les jeunes générations d'une tension face à l'avenir? Quoi qu'il en soit, il est clair que le mouvement rave exerce un incroyable pouvoir de fascination sur bon nombre d'adolescents et de jeunes adultes et que, pour beaucoup, il représente le laboratoire où l'on expérimente le présent, la transgression et où l'«échange» reprend momentanément son sens, indistinctement de sa forme économique et professionnelle généralisée dans les sociétés contemporaines.

Dans notre vie quotidienne, l'importance de la technique et des technologies atteint des sommets inégalés. Intensification d'un contexte proprement moderne, les technologies sont encore aujourd'hui synonymes de dépassement et de progrès. Cependant, elles peuvent également apparaître comme agent de séparation et d'individualisation du tissu social, autant que comme l'instrument d'une culture marchande recherchant une émancipation continuelle. Le mouvement rave, sur ce plan, se révèle de nature assez paradoxale. En effet, autant ces rassemblements trouvent leur pertinence en marge d'un système social dominant, autant ils puisent dans la technologie l'énergie nécessaire afin de mettre au monde de nouveaux types d'agrégation sociale de signification. Ainsi, il est possible d'assister à la convergence d'une culture technique et d'une énergie festive innovatrice ${ }^{8}$. Phénomène récent et précurseur, le mouvement rave est donc à ce titre un bon exemple des manifestations spontanées à travers lesquelles certains éléments 
de l'activité sociale se voient réappropriés sans objectif particulier, mais ayant comme résultat de donner lieu à l'émergence de nouveaux modes de sensibilité. Finalement, au moment même où s'intensifie une ère de communication et d'information, le mouvement rave (et son fil conducteur qu'est la musique techno) donne tout son sens à la maxime de Marshall McLuhan soulignant que «le médium est le message". En effet, dénuée de parole ou de message, mais non moins englobante et chargée d'identités, la techno démontre que pour les raveurs, le sens réside uniquement dans l'action communicationnelle elle-même, ainsi que dans les liens qui y sont générés?.

\section{Retour au tribalisme et effervescence rave}

Comme nous l'avons mentionné précédemment, nos sociétés subissent des transformations qui font écho au plus profond de l'individu, jusque dans son rapport intime au sacré. Le mouvement rave confirme ces transformations, d'autant plus qu'il se traduit en des lieux privilégiés d'effervescence et de resserrement de liens sociaux. La pensée de Michel Maffesoli nous permet d'ailleurs à cet égard de rendre compte du fait que le phénomène rave suit le rythme du contexte actuel. En effet, pour Maffesoli, le contexte de postmodernité est celui de nos sociétés de masse : mégapoles, pluralisme, polythéisme des valeurs, faillite du mythe du progrès, immersion dans un présent instantané, passage d'une société civile responsable à une masse désabusée face à la chose publique, etc. Toutefois, l'auteur avance que «le dynamisme social n'emprunte plus les voies propres à la modernité10", et que l'on assiste depuis quelques décennies à une redynamisation du lien social par la mise en place d'un «néo-tribalisme». Les sociétés contemporaines relègueraient peu à peu à la marge les anciennes formes politico-institutionnelles d'intégration du social relative à la modernité au profit d'une multitude de nouvelles communautés qu'il qualifie de «tribus". À l'instant où l'individualisme semblait avoir atteint son paroxysme, Maffesoli met en lumière l'apparition d'un réseau implicite de tribus locales ou de "communautés de sensations" mouvantes, éphémères et multiformes (sectes, gangs de quartiers, clubs de rencontres, clubs sportifs, associations, etc.) par lesquelles l'individu serait appelé vers l'autre dans un but de réalisation d'expériences communes et de vie "affectuelle»11. II s'établit donc une véritable sacralité des rapports sociaux, ce que Maffesoli, à l'instar de Durkheim, appelle le "divin social». Bien entendu, si déplacement du religieux il y a, il n'est pas étonnant, suivant cette thèse, qu'il se manifeste dans des formes particulièrement tribales, notamment dans le mouvement rave. 


\section{Rituel contemporain}

L'homme fait corps avec le rituel et le rituel fait corps avec l'homme

Denis Jeffrey

À travers les manifestations raves, comme dans d'autres sphères du social, se dessinent les structures et les fonctions des rituels d'autrefois. Plusieurs auteursxii, devant le déplacement et les mutations du religieux, furent amenés à parler de sécularisation des rites. En effet, on retrouve une multitude de rites dorénavant disséminés au sein de la culture contemporaine et empreints de significations nouvelles. Toutefois, il n'en demeure pas moins que les rites, bien qu'ayant changé de forme, restent une dimension essentielle de la vie des hommes par laquelle ils peuvent créer du sens et célébrer la mémoire collective ${ }^{13}$.

Dans les sociétés contemporaines, le rituel tend à prendre de nouvelles formes quant à la manière dont il est vécu. Le Breton a défini en quoi le rituel s'éloigne aujourd'hui de sa nature traditionnelle. En effet, davantage intimes, détachés de la mémoire collective et n'ayant de signification que pour l'individu ou le petit groupe qui les vit, les rituels modernes ne sont d'ailleurs pas toujours conformes aux normes sociales établies par la communauté. À cet effet, ils sont généralement vécus dans la clandestinité par les jeunes générations. Bien qu'il ne s'agisse plus d'un passage obligé et générateur de valeurs sociales communes, les rituels ne sont pas moins repérables statistiquement par une succession d'actes ou de pratiques à la base de certains phénomènes sociaux (pensons par exemple au tatouage, au piercing ou encore aux initiations universitaires, pour ne mentionner que ces formes nouvelles de rituels).

Dans les hangars, sortes d'églises dans lesquelles un retour à la foi est possible, le rituel du rave joue un rôle prépondérant au niveau de la signification que l'acteur accorde au monde qui l'entoure. En effet, même si l'exercice religieux du rite a subi des transformations morphologiques en certains points, ces fonctions sont cependant restées les mêmes. Entre autres, d'hier à aujourd'hui, le rite a toujours su répondre à des besoins de création de sens, de pacification de l'angoisse et de la violence, de maintien du lien social, de confirmation de l'appartenance identitaire et de réalisation de transcendance. L'observateur sera en mesure d'identifier tous ces éléments au sein des manifestations raves; hormis qu'une des principales 
caractéristiques du rave en tant que rituel est que ce dernier n'est pas véritablement rattaché à un récit fondateur, contrairement aux rites traditionnels. Le concept de "rituel sauvage», avancé par Roger Bastide acquiert donc ici toute sa pertinence. En effet, le concept de «rite sauvage» met davantage l'accent sur le caractère spontané des conduites qui animent les rituels contemporains ${ }^{14}$. D'ailleurs, à juste titre, la célébration rave n'est-elle pas celle du moment présent? Les manifestations raves, par leur attribut principal qui consiste à façonner la sensibilité et à entretenir les ardeurs collectives, soulignent la nécessité symbolique du regroupement et du partage de moments communs d'intensité.

\section{Quête de sens}

La prégnance de la religion avait jadis largement contribué, de par sa nature, à fournir aux hommes les clés de la vérité. Aujourd'hui, il peut s'avérer complexe de donner un sens à la vie et d'en trouver les limites tant l'autonomie et les conditions de possibilités semblent infinies. Face au flou symbolique d'une société qui n'offre plus de réponses aux grandes questions de l'humanité, face à la monotonie d'une vie circulaire, le mouvement rave serait-il vécu comme une échappatoire à l'aliénation croissante dans laquelle l'homme de la postmodernité serait plongé? Cette question semble intéressante, d'autant plus qu'elle amène à considérer les rassemblements rave comme des zones franches situées à l'extérieur de la routine du système social dominant. Soulignons ici l'apport des théories d'Érik Cohen. En effet, bien que son travail se soit élaboré autour d'une phénoménologie de l'expérience touristique, ainsi qu'autour du concept de centre-périphérie, il est possible de voir des analogies entre les deux phénomènes. En effet, selon les principes de l'analyse de Cohen, c'est dans un mode "expérimental ${ }^{15}$ " que le raveur trouvera un certain réconfort en faisant l'expérience esthétique de l'authenticité des autres. Dans la périphérie de son centre symbolique d'appartenance ${ }^{16}$, en marge des expériences insatisfaisantes du monde commun, il collaborera à l'élaboration d'un centre symbolique éphémère, une sorte de "Techno-cosmos 17" par lequel tout reprendra son sens; il deviendra, comme le souligne Ghislain Fournier : «le participant actif d'une réalité qui le dépasse, l'aventurier des obscurs chemins de l'extase ${ }^{18}$ ". Cette aventure, sorte de pèlerinage moderne vers un centre symbolique réinventé en périphérie du monde, participera donc à sa façon à la réalisation d'une quête vers la ressemblance et l'appartenance. 
II existe plusieurs façons d'aborder le phénomène rave en tant que quête de sens. Hormis l'apport du concept de centre symbolique, permettant de comprendre comment les rassemblements raves constituent une sorte de fuite, - ou du moins une sorte de pèlerinage vers une vérité tant convoitée - on peut également considérer ce phénomène contemporain comme une fusion entre quête de sens et quête des sens. Effectivement, comme le souligne David Le Breton dans l'ouvrage intitulé Fabrication individuelle de sens et jeux avec la mort : Les jeunes générations, c'est dorénavant par le rapport physique au monde que l'on tentera de trouver les repères et le sens nécessaire à la compréhension du monde. C'est dans un corps à corps avec les limites de ses sens que l'individu confirmera désormais son existence: le réel tend à remplacer le symbolique dans la construction d'un sens à son univers ${ }^{19}$. Les rassemblements raves, par les sensations fortes, plaisirs intenses et contacts qui s'y dégagent, répondent à un besoin largement répandu chez les adolescents et les jeunes adultes, besoin qui, d'autant plus, n'est pas satisfait dans les sphères de la vie ordinaire. Ainsi, les manifestations raves prennent en quelque sorte la forme de rituels hédonistes 20. Selon Denis Jeffrey, les raves, comme bon nombre de rituels contemporains qui impliquent une jouissance intense et immédiate, canalisent les forces de vie excédentaires, celles pouvant provoquer violence et transgressions. En fait, les raves libèrent l'énergie interdite pour le simple plaisir de "sentir intensément la vie couler dans son corps $^{21}$ ».

\section{Drogue et transcendance}

L'usage de drogue est une partie intégrante de l'expérience rave. Ecstasy 22, speed ou GHB, les drogues raves ont comme effets communs d'amplifier les sens et de permettre au corps de danser sans relâche pour des périodes pouvant aller jusqu'à plus de douze heures. L'angle d'analyse que nous avons adopté jusqu'à maintenant incite à relever un point intéressant à ce propos. En effet, l'importance des drogues dans l'altération de la conscience éveillée et dans l'impression d'une expérience transcendante, surnaturelle et sans égale est un aspect non négligeable qui vient ajouter aux manifestations raves un caractère proprement religieux. L'utilisation de drogues donne accès (ou l'impression d'accès) à une réalité idéale depuis longtemps recherchée ${ }^{23}$. De plus, les substances sont consommées au début du rituel et détermineront l'intensité et la durée de celui-ci. À travers la culture de la beat generation, dont Jack 
Kerouac a fait une description étayée dans Sur la route 24 , on retrouve une forme similaire d'usage de drogues, notamment du LSD, afin d'accéder à une spiritualité nouvelle. Certaines sectes utilisent également les drogues, autant pour altérer les facultés rationnelles et critiques des disciples que pour faire apparaître comme vraisemblables certaines manifestations. II ne fait aucun doute que sans la consommation systématique de drogue dans les manifestations raves, l'expérience vécue lors de ces événements serait assez différente, mais elle conserverait sans doute encore un intérêt religiologique, à l'instar de celle éprouvée lors des grands rassemblements sportifs ou artistiques ${ }^{25}$.

\section{Conclusion}

L'émergence et la popularité des rassemblements raves n'est pas un phénomène isolé, bien au contraire. II s'inscrit dans une vaste transformation sociétale où le religieux tend à se déplacer en des lieux nouveaux et inédits. Témoignant du besoin de donner sens aux expériences du monde, de symboliser et de ritualiser le moment présent, ainsi que de sacraliser la vie en commun, ne serait-ce qu'un instant, en marge des impératifs économiques et professionnels des sociétés contemporaines, les raves sont, pour le sociologue des phénomènes religieux, un exemple nécessaire à la compréhension d'une réalité en pleine mutation.

Vécu et exprimé, le contexte social actuel semble a priori déroutant pour plusieurs jeunes. De même, plusieurs problématiques tels que le décrochage scolaire ou le suicide sont souvent mis de l'avant afin de dénoncer les principaux fléaux qui en résultent. Pourtant, sous nos yeux, en des lieux et circonstances insoupçonnés, un nouveau monde de significations prend forme, avec toute la spontanéité et l'intensité propres à la jeunesse. L'étude des raves, compris alors comme réponse à un besoin de signification au départ inassouvi, bouleverse notre conception première des événements festifs de ce genre. Face aux discours désapprobateurs véhiculés par les médias ou par l'opinion populaire, une telle ouverture ne peut être que bienfaitrice.

D'autre part, si les hypothèses abordées précédemment ne reflètent qu'un des nombreux visages des mutations du sacré et de la pensée symbolique du monde contemporain, il serait intéressant de poursuivre qualitativement l'étude par une série d'entrevues afin de favoriser une meilleure compréhension de la conception symbolique 
des jeunes d'aujourd'hui. Si, il n'y a pas si longtemps, nous proclamions "la mort des religions ${ }^{26 », ~ l e ~ r e l i g i e u x ~ q u a n t ~ a ̀ ~ l u i ~ p o u r r a i t ~}$ bien être indissociable de la condition humaine, et ce, même aujourd'hui, en de multiples formes inattendues.

\section{Pierre-Luc Lortie Sociologie, université du Québec à Montréal}

1. François Gauthier et Guy Ménard (dir.), "Technoritualités : Religiosité rave», Religiologiques, no 24 (automne 2001), Montréal, Université du Québec à Montréal.

2. Paul Tillich, dans Danièle Hervieu-Léger, La religion pour mémoire.Paris, Éditions du cerf, coll. Sciences humaines et religions, 1993, p. 61.

3. Paul Tillich, op. cit., p. 105

4. Roger Bastide, dans Guy Ménard, "Les déplacements du sacré et du religieux", Érudit, (page consultée le 27 novembre 2003), (En ligne), Adresse URL : http://www.erudit.org/livre/larouchej/2001/livrel4 div23.htm

5. Guy Ménard, op.cit.

6. François Gauthier et Guy Ménard, op.cit., p. 177-178.

7. Michel Gaillot, "Les raves, «part maudite» des sociétés contemporaines», Sociétés, no 72, 2001-2002, p. 47-48.

8. Ibid., p. 49.

9. Ibid., p. 47-48.

10. Michel Maffesoli, Le temps des tribus, Paris, Méridiens Klincksieck, 1988, p. 11.

11. Ibid., p. 210-214, 217.

12. Notamment Raymond Lemieux et Raymond Brodeur cité dans Denis Jeffrey. «Les rituels contemporains", Érudit, (page consultée le 27 novembre 2003), (En ligne), Adresse URL : http://www.erudit.org/livre/larouchej/2001/livre/4 div24.htm

13. Denis Jeffrey, op.cit.

14. Ibid.

15. Concept emprunté à Erik Cohen, "A Phenomenology of Tourist Experiences", Sociology, vol.13, 1979, p.179-201 ayant pour objet une phénoménologie de l'expérience touristique.

16. Ibid. p. 182-183.

17. Michel Gaillot, op.cit. p. 51-55.

18. Ghislain Fournier, «Rave, intensité et quête de sens», Technoritualités : Religiosité rave, p. 77.

19. David Le Breton, «Fabrication individuelle de sens et jeux avec la mort: les jeunes générations", Revue internationale d'action communautaire, no 29/69, 1993, p. 128-129. 
20. Conception selon laquelle le plaisir devient la norme de toute conduite (même morale), l'hédonisme renvoie à la recherche du plaisir sous toutes ses formes. Louis-Marie Morfaux, "Plaisir" et "Hédonisme», Vocabulaire de la philosophie et des sciences humaines, Paris, Armand Colin, 1980.

21. Denis Jeffrey, "Le rave : mise en scène d'une jouissance transgressive». In Technoritualités : Religiosité rave, p. 135.

22. D'après l'Agence française de sécurité sanitaire des produits de santé, l'ecstasy (MDMA) est reconnu pour ses propriétés psychostimulantes et hallucinogènes et ses effets sont sujets à changement selon la dose, les attentes de l'utilisateur, le lieu, etc. Son utilisation soumet l'individu à des risques de troubles psychologiques tels que l'anxiété, la dépression et des épisodes psychotiques.

23. François Gauthier et Guy Ménard, op.cit., p. 168

24. Jack Kerouac, Sur la route, Paris, Gallimard, 1960.

25. Grace Davie, citée par Jean Paul Willaime, "Le croire, l'acteur et le chercheur : Introduction au dossier Croire et modernité", Arch. de Sc. Soc. des Rel., no 81, 1993, p. 12 et Guy Ménard, "Les déplacements du sacré et du religieux", Érudit, (page consulté le 27 novembre 2003), (En ligne), Adresse URL : http://www.erudit.org/livre/larouchej/2001/ivrel4 div23.htm

26. Danièle Hervieu-Léger, 1996. "Productions religieuses de la modernité : Les phénomènes du croire dans les sociétés modernes", Religion, sécularisation, modernité : Les expériences francophones en Amérique du Nord, Sainte-Foy, Les Presses de l'Université Laval, p. 37. 